\title{
Radical Resection for Second EGFR-mutated Primary Lung Cancer Following Immune Checkpoint Inhibitor Monotherapy for Stage IV Lung Adenocarcinoma
}

\author{
Ryota Horibe $^{1}$, Taku Hatakeyama ${ }^{1}$, Tatsuru Ishikawa ${ }^{2}$, Takeyuki Sawai ${ }^{3}$, Midori Hashimoto ${ }^{1}$, \\ Hiromitsu Domen ${ }^{4}$, Yasunari Takakuwa ${ }^{5}$, Masaaki Satoh ${ }^{5}$ and Kaoru Nishiyama ${ }^{1}$
}

\begin{abstract}
:
A 78-year-old woman with multiple lung nodules, epithelial growth factor receptor (EGFR) exon 20 insertion mutations, and diagnosed with advanced lung adenocarcinoma (cT4N3M1a, stage IVA), was referred to our hospital. She received immune checkpoint inhibitor (ICI) therapy. The therapy showed remarkable antitumor effects; only a single nodule remained in the right upper lobe. The nodule was diagnosed as adenocarcinoma through a biopsy. We subsequently performed right upper lobectomy for multiple primary lung cancer (MPLC). The surgical specimen contained EGFR exon 19 deletion mutations and not exon 20 insertion mutations.
\end{abstract}

Key words: EGFR-mutated lung adenocarcinoma, exon 20 insertion, pembrolizumab, multiple primary lung cancer

(Intern Med Advance Publication)

(DOI: 10.2169/internalmedicine.6385-20)

\section{Introduction}

Lung cancer is the most common cause of cancer-related deaths worldwide (1). In Japan, approximately $30 \%$ of lung cancers are diagnosed at stage IV (2). In recent years, immune checkpoint inhibitors (ICIs) have become the treatment of choice for advanced non-small-cell lung cancers (NSCLCs). Pembrolizumab has been shown to be effective in the treatment of NSCLCs with a high expression of the programmed death-ligand 1 (PD-L1) (3). When administered with platinum-doublet chemotherapy, pembrolizumab has been shown to be also effective in NSCLCs with low PD-L1 expression (4).

Because ICI therapy has improved the prognosis of patients with advanced NSCLC, the treatment for residual lesions following ICI therapy is expected to increase in the future. We herein the remarkable antitumor effect of ICI ther- apy on tissues with EGFR-mutated (exon 20 insertion mutations) stage IV lung adenocarcinoma. We also successfully resected a nodule thought to be MPCL.

\section{Case Report}

A 78-year-old woman was referred to our hospital for multiple lung nodules. No other personal medical history was reported, except for an episode of goiter three years earlier. She had no history of malignancy or collagen disease. She was a never-smoker.

Chest and abdominal computed tomography (CT) showed a 55-mm mass on the left upper lobe, multiple nodules in both lungs, and multiple enlarged mediastinal lymph nodes (Fig. 1a-c). Laboratory examinations revealed elevated levels of cytokeratin-19 fragments (CYFRA $4.8 \mathrm{ng} / \mathrm{mL}$ ) and normal levels of CEA, CA19-9, SLX, and NSE. A bronchoscopic biopsy was performed on the left upper lobe. The pa-

\footnotetext{
${ }^{1}$ Department of Respiratory Medicine, NTT-East Corporation Sapporo Medical Center, Japan, ${ }^{2}$ Department of Respiratory Medicine and Allergology, Sapporo Medical University School of Medicine, Japan, ${ }^{3}$ Department of Respiratory Medicine, Hakodate City General Hospital, Japan, ${ }^{4}$ Department of Surgery, NTT-East Corporation Sapporo Medical Center, Japan and ${ }^{5}$ Department of Pathology, NTT-East Corporation Sapporo Medical Center, Japan

Received: September 28, 2020; Accepted: June 28, 2021; Advance Publication by J-STAGE: August 13, 2021

Correspondence to Dr. Ryota Horibe, r_horibe_57@yahoo.co.jp
} 


\section{Before pembrolizumab therapy}
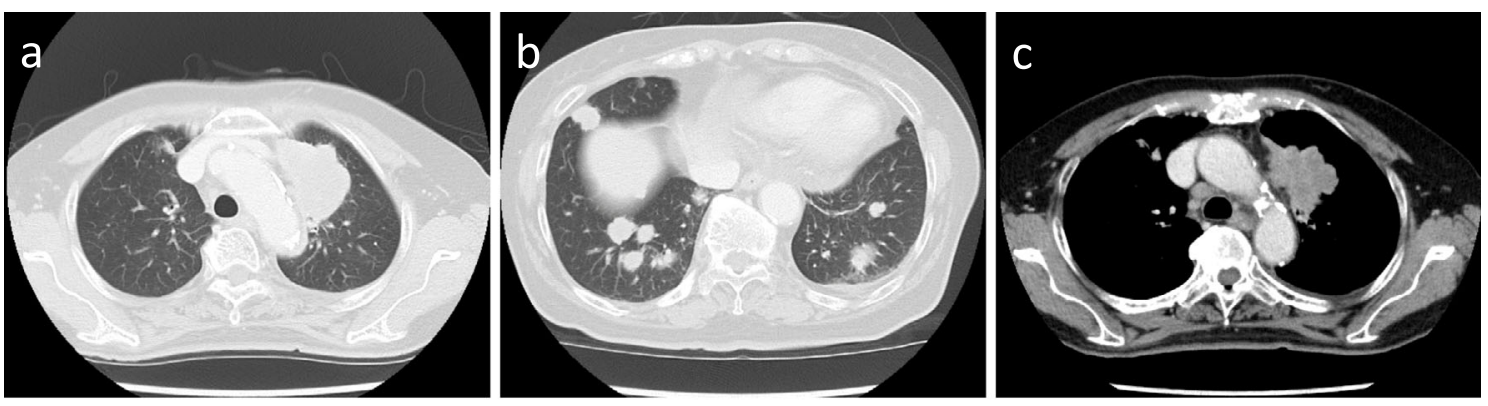

After pembrolizumab therapy
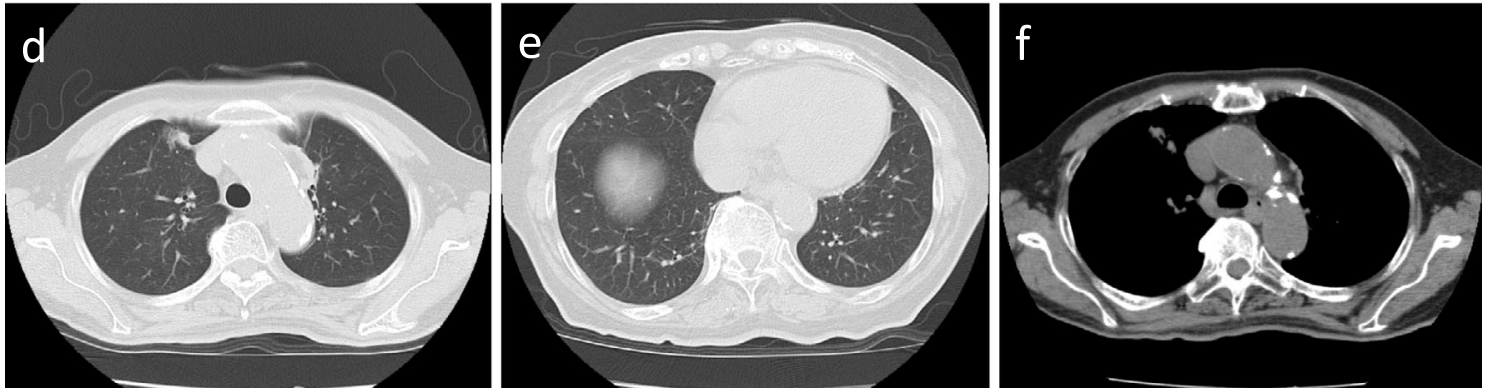

Figure 1. Chest CT. (a, b) Mass in the left upper lobe and many nodules in both lungs. (c) Multiple enlarged mediastinal lymph nodes. (d, e, f) Mass in the left upper lobe, multiple nodules in both lungs, and multiple mediastinal lymph nodes that were reduced following pembrolizumab therapy.

thologic diagnosis of the biopsied specimens was poorly differentiated lung adenocarcinoma (Fig. 2a). The tumor showed a high PD-L1 expression (tumor proportion score [TPS]: 95\%) (Fig. 2b). In addition, EGFR exon 20 insertion mutations were detected. She was ultimately diagnosed with lung adenocarcinoma with cT4N3M1a (PUL), stage IVA.

Although the tumor was EGFR-mutated adenocarcinoma, ICI therapy was used because of the EGFR exon 20 insertion mutations and high expression of PD-L1. The patient was administered pembrolizumab (200 mg/body, every 3 weeks). After 17 cycles, CT was performed that showed a reduction in the size of all lymph nodes and disappearance of the lung nodules (Fig. 1d-f), except for one nodule that remained in the right upper lobe (Fig. 3a, b).

The therapeutic effect was evaluated as a partial response based on the response evaluation criteria in solid tumors (RECIST); however, the patient discontinued the pembrolizumab treatment because of nonbacterial cystitis, an immune-related adverse event. After five months of pembrolizumab withdrawal, chest CT was performed that showed enlargement of the remaining nodule in the right upper lobe (Fig. 3c). Fluorodeoxyglucose-positron emission tomography/CT was performed for the further evaluation, revealing an increased FDG uptake in the nodule only (Fig. 4a, b). A bronchofiberscope biopsy was performed, and the nodule was diagnosed as lung adenocarcinoma. No lymph node metastasis or systemic metastasis was observed. In addition, the right upper nodule had an unclear border, unlike the other lung nodules. Therefore, we considered the possibility of MPLC and performed radical resection of the right upper lobe.

Initially, video-assisted thoracic surgery (VATS) lung resection was performed. However, thoracotomy was required because of the presence of intense adhesions around the main pulmonary artery at the hilum and a lymph node at the hilum and mediastinum. The operative time was $223 \mathrm{~min}$, and the estimated blood loss was $30 \mathrm{~mL}$. She received no intraoperative blood transfusion and was hospitalized for nine days after the operation.

After the operation, EGFR exon 19 deletion mutations (TPS $<1 \%$ ) were identified on the surgical specimen and diagnosed as MPLC (Fig. 2c, d). The patient received tegafururacil treatment as adjuvant therapy following the operation. No recurrence was observed in the patient one year after right upper lobectomy.

\section{Discussion}

ICI therapy has revolutionized the ever-changing therapeutic landscape of some advanced lung cancers, such as NSCLCs. In the present case, ICI therapy was effective in treating the EGFR-mutated (exon 20 insertion mutations) lung adenocarcinoma. EGFR exon 20 insertion mutations account for up to $4 \%-10 \%$ of EGFR mutations in lung cancers (5). As opposed to other EGFR-mutated lung cancers, tumors with exon 20 insertion mutations respond poorly to EGFR tyrosine kinase inhibitors (6). Such treatments are also not effective in EGFR-mutated cancers with high PD-L 1 expression (7). However, several studies have shown opposite results. Masuda et al. reported that PD-L1 expression is 


\section{The left upper lobe specimen by biopsy}
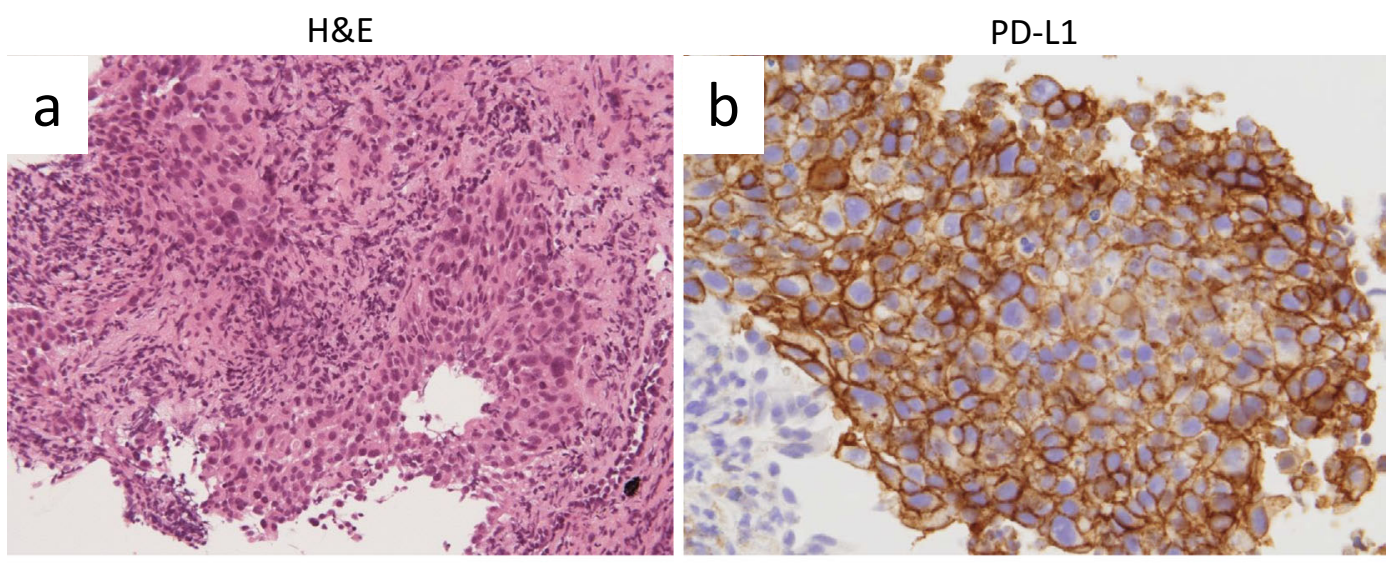

The right upper lobe specimen by lobectomy

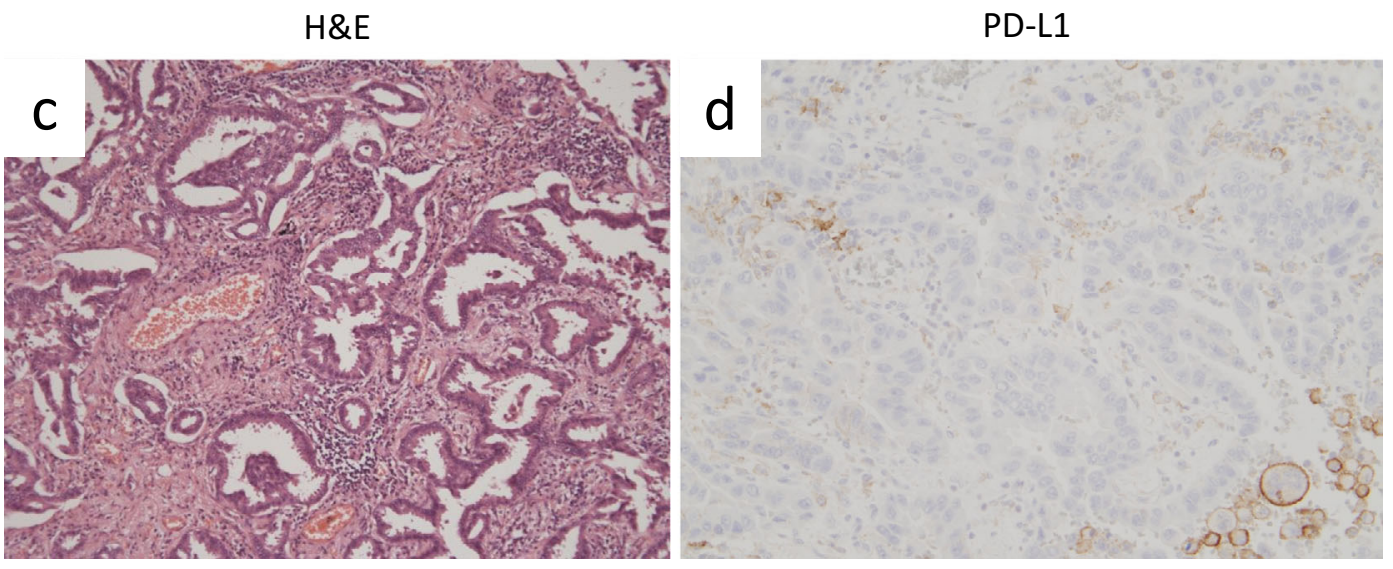

Figure 2. Pathological findings. The left upper lobe specimen by biopsy: (a) Hematoxylin and Eosin (H\&E) staining, $\times 200$, (b) PD-L1 staining, $\times 400$, The right upper lobe specimen by lobectomy: (c) H\&E staining, $\times 200,($ d) PD-L1 staining, $\times 400$. PD-L1 staining was used the $22 \mathrm{C} 3$ antibody.

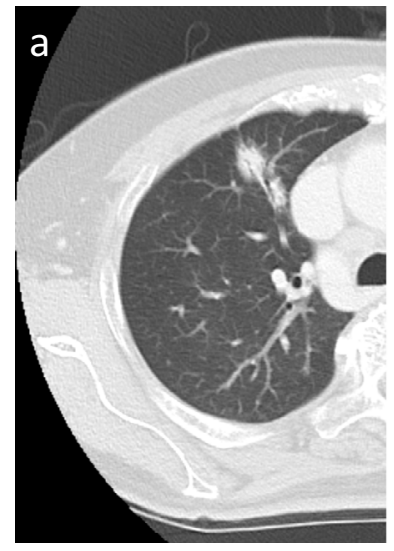

Before pembrolizumab therapy

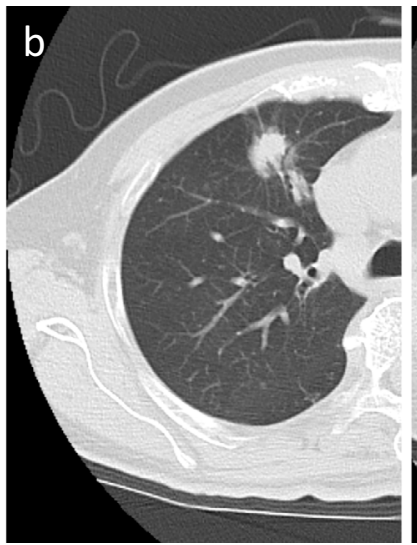

At time of cessation of pembrolizumab therapy

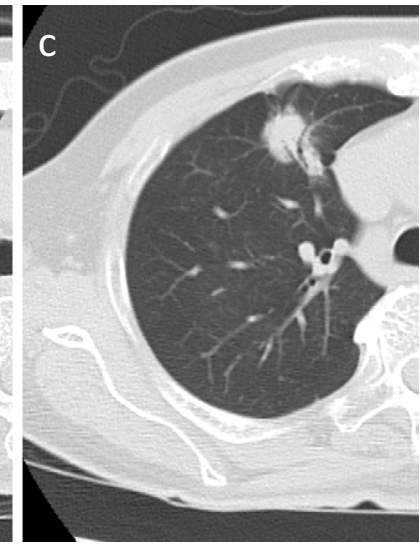

Five months after ending pembrolizumab therapy

Figure 3. Chest CT. (a, b, c) Nodule in the right upper lobe grew slowly.

associated with the efficacy of ICIs in patients with EGFR mutations. In the high-PD-L1-expression group, the objective response rate (ORR) was $29.4 \%$, whereas it was $0 \%$ in the low-PD-L1-expression group (8). Miyawaki et al. reported that the ORR of pembrolizumab for uncommon
EGFR-mutated NSCLCs was 60\% (9). Regarding EGFR exon 20 insertion mutations, several studies have reported on the antitumor effect of ICI therapy $(10,11)$. Interestingly, the subtypes of EGFR mutation and the PD-L1 expression differed between left and right upper tumors; in addition, the 


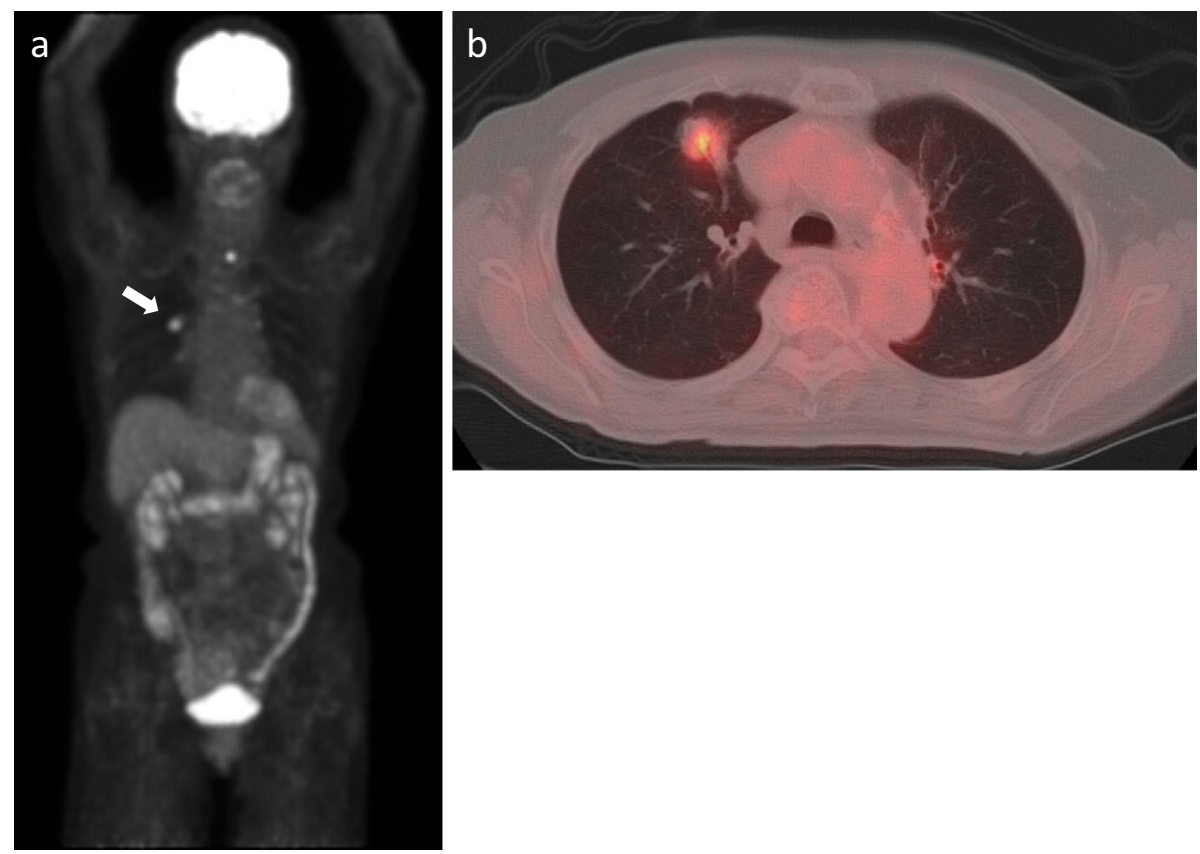

Figure 4. (a, b) A FDG-PET/CT reveals the FDG uptake by the remaining nodule in the right upper lobe (arrow).

response to ICI therapy was also different between these tumors. Although several biomarkers (such as the PD-L1 expression, tumor mutation burden, and tumor-infiltrating immune cells) have been investigated to determine the ICI efficacy, definitive prognostic factors are still not known. Further studies are thus needed to evaluate these factors.

There are no generally recognized guidelines for MPLC, although some diagnostic criteria are widely used in clinical investigations (12-14). According to these criteria, MPLCs can be divided into two groups: synchronous MPLC and metachronous MPLC. These criteria show that the following findings favor the synchronous MPLC: differences in the radiographic appearance, metabolic uptake, and growth rates and an absence in lymphatic or systemic metastases even with the same histology. In our patient, the nodule in the right upper lobe showed an unclear border with spiculation, unlike other lung nodules in retrospect. Furthermore, no lymphatic or systemic metastasis was observed. We therefore diagnosed the nodule as MPLC before the operation. Some reports indicated that positron emission tomography (PET)CT could distinguish between MPLC and intrapulmonary metastasis (IM) based on the difference in the maximum standardized uptake value $\left(\mathrm{SUV}_{\text {max }}\right)(15,16)$. The difference in the $\mathrm{SUV}_{\max }$ between lesions was significantly higher in patients with MPLC than in those with IM. However, we did not perform PET-CT before the first-line chemotherapy. If we had performed PET-CT, it might have helped us diagnose the MPLC before the operation by comparing the SU$\mathrm{V}_{\max }$ values of the left upper tumor and the right upper nodule.

MPLC has been detected in approximately $0.5 \%-0.97 \%$ of patients with lung cancer at the diagnosis. It has been estimated that the risk of developing second lung cancer in pa- tients who survived resection of an NSCLC-affected tissue is approximately $1 \%-2 \%$ per year (17-19). ICI therapy has become the first-line therapy for most NSCLCs. In addition, several clinical trials of neoadjuvant and adjuvant ICI therapies are also being conducted. We thus expect that, as the use of ICI therapy increases, the consideration of pulmonary resections for metachronous MPLC or oligometastasis following ICI therapy will increase as well.

While ICI therapy is considered promising for the treatment of NSCLCs, few reports are available concerning pulmonary resection in lung cancers following ICI therapy. Chaft et al. were the first to report five cases with advanced NSCLCs for which pulmonary resection was performed for persistent local disease following ICI therapy. They showed that pulmonary resection was feasible without any major postoperative complications (20). Bott et al. reported 19 cases in which pulmonary resection was performed following ICI therapy for unresectable lesions, such as distant metastatic disease or extensive mediastinal lymphadenopathy. Although dense adhesions at either the hilum or chest wall existed in a few cases, they concluded that surgical resection following ICI therapy is apparently safe, with mostly minor complications. The outcomes from these cases were encouraging, as they showed reasonable survival rates during the short-interval follow-up (21). Forde et al. reported that neoadjuvant therapy with nivolumab in surgically resectable disease, i.e. early NSCLC, was associated with few side effects. As a result, lung surgery was not delayed, and excess postoperative complications were not observed (22). In our case, thoracotomy was required because of intense adhesions at the hilum of the lung; however, no postoperative complications occurred, and the duration of hospitalization was also short. It is thus important to pay adequate attention to 
patients following ICI therapy in order to detect any recurrence of tumors at an early stage and consider radical resection if possible.

In conclusion, this report showed that a patient with EGFR-mutated advanced lung adenocarcinoma, who received ICI therapy as the primary treatment was able to undergo radical resection of the residual disease because of the remarkable antitumor effect.

The authors state that they have no Conflict of Interest (COI).

\section{Acknowledgement}

We would like to thank WORDVICE (https://wordvice.com) for the English language editing.

\section{References}

1. Barta JA, Powell CA, Wisnivesky JP. Global Epidemiology of Lung Cancer. Ann Glob Health 85 (8): 2019.

2. Cancer information service, National cancer center, japan. annual report of hospital-based cancer registries.

3. Reck M, Rodríguez-Abreu D, Robinson AG, et al. Updated Analysis of KEYNOTE-024: Pembrolizumab Versus Platinum-Based Chemotherapy for Advanced Non-Small-Cell Lung Cancer With PD-L1 Tumor Proportion Score of 50\% or Greater. J Clin Oncol 37: 537-546, 2019.

4. Gandhi L, Rodríguez-Abreu D, Gadgeel S, et al. Pembrolizumab plus Chemotherapy in Metastatic Non-Small-Cell Lung Cancer. N Engl J Med 378: 2078-2092, 2018.

5. Vyse S, Huang PH. Targeting EGFR exon 20 insertion mutations in non-small cell lung cancer. Signal Transduct Target Ther 4 (5): 2019.

6. Yang JC, Sequist LV, Geater SL, et al. Clinical activity of afatinib in patients with advanced non-small-cell lung cancer harbouring uncommon EGFR mutations: a combined post-hoc analysis of LUX-Lung 2, LUX-Lung 3, and LUX-Lung 6. Lancet Oncol 16: 830-838, 2015.

7. Lisberg A, Cummings A, Goldman JW, et al. A Phase II Study of Pembrolizumab in EGFR-Mutant, PD-L1+, Tyrosine Kinase Inhibitor Naïve Patients With Advanced NSCLC. J Thorac Oncol 13: 1138-1145, 2018.

8. Masuda K, Horinouchi H, Tanaka M, et al. Efficacy of anti-PD-1 antibodies in NSCLC patients with an EGFR mutation and high PD-L1 expression. J Cancer Res Clin Oncol 147: 245-251, 2021.
9. Miyawaki E, Murakami H, Mori K, et al. PD-L1 expression and response to pembrolizumab in patients with EGFR-mutant nonsmall cell lung cancer. Jpn J Clin Oncol 50: 617-622, 2020.

10. Takeda M, Sakai K, Hayashi $\mathrm{H}$, et al. Clinical characteristics of non-small cell lung cancer harboring mutations in exon 20 of EGFR or HER2. Oncotarget 9: 21132-21140, 2018.

11. Lau SCM, Fares AF, Le LW, et al. Subtypes of EGFR- and HER 2-Mutant Metastatic NSCLC Influence Response to Immune Checkpoint Inhibitors. Clin Lung Cancer 7: S1525-7304(21) 00003-6, 2021.

12. Martini N, Melamed MR. Multiple primary lung cancers. J Thorac Cardiovasc Surg 70: 606-612, 1975.

13. Kozower BD, Larner JM, Detterbeck FC, Jones DR. Special treatment issues in non-small cell lung cancer: Diagnosis and management of lung cancer, 3rd ed: American College of Chest Physicians evidence-based clinical practice guidelines. Chest 143: e369S-e399S, 2013.

14. Rami-Porta RAH, Travis WD, Rusch VW. AJCC Cancer Staging Manual. 8 ed. Springer International Publishing, France, 2017.

15. Dijkman BG, Schuurbiers OC, Vriens D, et al. The role of (18)FFDG PET in the differentiation between lung metastases and synchronous second primary lung tumours. Eur J Nucl Med Mol Imaging 37: 2037-2047, 2010.

16. Liu Y, Tang Y, Xue Z, et al. UVmax Ratio on PET/CT May Differentiate Between Lung Metastases and Synchronous Multiple Primary Lung Cancer. Acad Radiol 27: 618-623, 2020.

17. M K Ferguson. Synchronous primary lung cancers. Chest 103: 398S-400S, 1993.

18. Martini N, Melamed MR. Multiple primary lung cancers. J Thorac Cardiovasc Surg 70: 606-612, 1975.

19. Johnson BE. Second lung cancers in patients after treatment for an initial lung cancer. J Natl Cancer Inst 90: 1335-1345, 1998.

20. Chaft JE, Hellmann MD, Velez MJ, Travis WD, Rusch VW. Initial Experience With Lung Cancer Resection After Treatment With TCell Checkpoint Inhibitors. Ann Thorac Surg 104: e217-e218, 2017.

21. Bott MJ, Cools-Lartigue J, Tan KS, et al. Safety and Feasibility of Lung Resection After Immunotherapy for Metastatic or Unresectable Tumors. Ann Thorac Surg 106: 178-183, 2018.

22. Forde PM, Chaft JE, Smith KN, Anagnostou V, et al. Neoadjuvant PD-1 Blockade in Resectable Lung Cancer. N Engl J Med 378: 1976-1986, 2018.

The Internal Medicine is an Open Access journal distributed under the Creative Commons Attribution-NonCommercial-NoDerivatives 4.0 International License. To view the details of this license, please visit (https://creativecommons.org/licenses/ by-nc-nd/4.0/).

(C) The Japanese Society of Internal Medicine Intern Med Advance Publication 